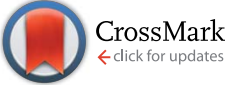

Cite this: Soft Matter, 2015, 11, 1547

\title{
Self-organized assemblies of colloidal particles obtained from an aligned chromonic liquid crystal dispersion
}

\author{
Natalie Zimmermann, ${ }^{a}$ Gisela Jünnemann-Held, ${ }^{a}$ Peter J. Collings ${ }^{\mathrm{bc}}$ \\ and Heinz-S. Kitzerow ${ }^{\star a}$
}

The behavior of mono-disperse colloidal particles in a chromonic liquid crystal was investigated. Poly(methyl methacrylate) spherical particles with three different functionalizations, with and without surface charges, were utilized in the nematic and columnar phases of disodium cromoglycate solutions. The nematic phase was completely aligned parallel to the glass substrates by a simple rubbing technique, and the columnar phase showed regions of similar alignment. The behavior of the colloidal particles in the chromonic liquid crystal depended critically on the functionality, with bromine functionalized particles not dispersing at all, and cationic trimethylammonium and epoxy functionalized particles dispersing well in the isotropic phase of the liquid crystal. At the transition to the nematic and especially the columnar phase, the colloidal particles were expelled into the remaining isotropic phase. Since the columnar phase grew in parallel ribbons, the colloidal particles ended up in chain-like assemblies. Such behavior opens the possibility of producing patterned assemblies of colloidal particles by taking advantage of the self-organized structure of chromonic liquid crystals.

Received 21st November 2014
Accepted 2nd January 2015

DOI: $10.1039 / c 4 s m 02579 b$

www.rsc.org/softmatter anisotropic properties, in particular, relatively large birefringence and large dichroism. One of the frequently used compounds in chromonic LCs is the azo dye sunset yellow (SSY), ${ }^{7}$ which is a common food additive. Another compound forming chromonic LCs is disodium cromoglycate (DSCG), which is used as an anti-asthmatic drug. ${ }^{8}$ Incorporation of other active molecules in the dye assemblies can also be applied to use chromonic LCs as drug delivery systems. ${ }^{9}$ The combination of biocompatibility and optical anisotropy can be applied for developing optical biosensors. ${ }^{\mathbf{1 0}}$ For example, Lavrentovich and coworkers demonstrated that the conformation of colloidal biomolecules can be optically detected by a chromonic LC, because these macromolecules create defects in the LC texture as soon as their size exceeds a critical threshold. ${ }^{10}$ The selforganization of chromonic LCs can also be utilized for the processing of technically important materials in a water-based environment. ${ }^{\mathbf{1 1}, \mathbf{1 2}}$ Getting a uniform alignment of chromonic LCs is one of the challenges impeding practical applications. Thus, recent studies are targeted to achieve uniform ${ }^{\mathbf{1 3 , 1 4}}$ and eventually even patterned ${ }^{\mathbf{1 5}}$ alignment. The latter purpose requires rather sophisticated techniques, such as nanoimprinting..$^{15}$ However, Collings and coworkers ${ }^{14}$ succeeded in finding a quite simple method of fabricating uniformly aligned chromonic LC samples through rubbing of the substrates with a fine abrasive foam. Based on this new alignment technique for chromonic LCs and motivated by previous work on monodisperse colloidal particles, ${ }^{\mathbf{1 6}}$ the present study aims to explore the behavior of colloidal particles in a uniformly aligned

\footnotetext{
${ }^{a}$ Department of Chemistry, University of Paderborn, 33098 Paderborn, Germany. E-mail: Heinz.Kitzerow@upb.de

${ }^{b}$ Department of Physics \& Astronomy, Swarthmore College, Swarthmore, Pennsylvania 19081, USA

${ }^{c}$ Department of Physics and Astronomy, University of Pennsylvania, Philadelphia, Pennsylvania 19014, USA
} 
chromonic LC. Using differently functionalized spherical particles based on poly(methyl methacrylate), PMMA, with and without surface charges, the mutual influence of the presence of such particles on the LC phase diagram and the influence of the self-organized LC structure on the spatial distribution of the particles are investigated. The role of surface functionalization of the PMMA spheres is explored by comparing three differently synthesized kinds of particles, bearing bromine atoms, quaternary ammonium ions, or epoxy groups on their surface. Aqueous solutions of DSCG where used as a host system. DSCG solutions have been extensively studied and show a representative morphology of different mesophases. ${ }^{17}$ Previous studies revealed the appearance of a nematic phase ( $\mathrm{N}$ phase), a columnar phase ( $\mathrm{M}$ phase), and regions of coexistence of one of these mesophases with the isotropic liquid phase (I) or with each other. X-ray studies ${ }^{17,18}$ have revealed the $\mathrm{N}$ phase to consist of short columnar stacks of single or double DSCG molecules, which grow with increasing DSCG concentration and assemble into a two dimensional hexagonal array of extended columns in the $\mathbf{M}$ phase. The formation of other proposed structures, including laterally extended, chimney-like assemblies $^{19}$ or thread-like assemblies, ${ }^{20}$ could not be confirmed by X-ray diffraction, ${ }^{\mathbf{1 8}}$ nor by scanning transmission X-ray microscopy (STXM), near edge X-ray absorption fine structure (NEXAFS) or birefringence measurements. ${ }^{21}$ Images of the molecular aggregates in chromonic liquid crystals have been recently obtained by transmission electron microscopy. ${ }^{22}$

\section{Results}

Chromonic LC samples were prepared on glass slides, the surface of which was uniaxially rubbed with a fine abrasive foam. Glass spacers with a diameter of $11 \mu \mathrm{m}$ were dispersed in two-component epoxy glue, which was used to assemble two of these substrates with a constant cell gap in between. Aqueous solutions of DSCG with concentrations between 12 and 25 wt $\%$ DSCG filled into these cells show very uniform textures. Fig. 1 shows the phase diagram of DSCG in water without particles. At room temperature, the nematic $(\mathrm{N})$ phase appears in the range of lower DSCG concentrations, the columnar (M) phase at larger DSCG concentrations. At elevated temperatures, there are broad regions of coexistence of the isotropic phase and a mesophase (either $\mathrm{N}$ or $\mathrm{M}$ ) owing to spontaneous separation into regions with lower and higher DSCG concentrations. This spinodal decomposition may lead to the appearance of DSCG-rich islands of the $\mathrm{M}$ phase which transform to the $\mathrm{N}$ phase on cooling. In a narrow range of concentrations close to $25 \mathrm{wt} \%$ DSCG, even a reentrant phase sequence $\mathrm{I}-(\mathrm{I}+\mathrm{M})-(\mathrm{I}+\mathrm{N})-\mathrm{N}-\mathrm{M}$ can be observed on cooling. These observations are in agreement with earlier studies. ${ }^{18}$

Intensity variations observed when the sample is rotated between crossed polarizers indicate a uniaxial alignment of the optical axis parallel to the substrate in both the $\mathrm{N}$ and $\mathrm{M}$ phases. This parallel alignment can be attributed to the rubbing procedure. Insertion of a wave plate ( $\lambda$-plate) at an azimuthal angle of $45^{\circ}$ indicates that the larger refractive index is observed if the plane of polarization (electric field of the light) is aligned

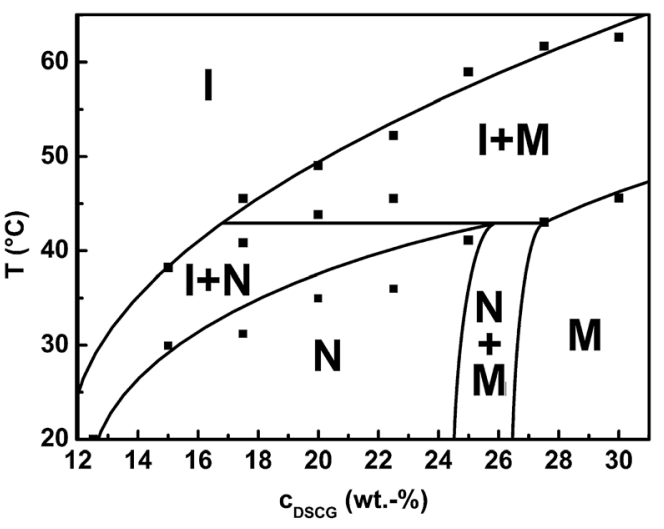

(a)

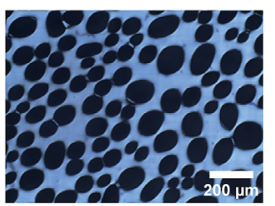

(b)

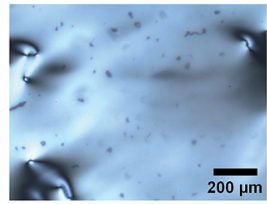

(d)

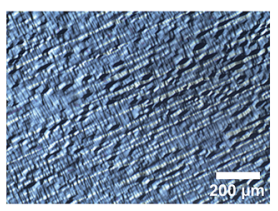

(c)

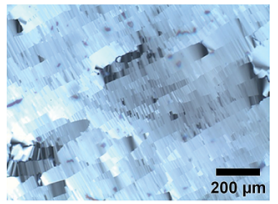

(e)
Fig. 1 (a) Phase diagram of DSCG in water. (b)-(e) Textures observed in the polarizing microscope with crossed polarizers: (b) coexistence of the nematic $(\mathrm{N})$ and the isotropic (I) phase, (c) coexistence of the columnar (M) and the isotropic (I) phase, (d) nematic (N) phase, (e) columnar (M) phase.

perpendicular to the rubbing direction. Since the $\mathbf{N}$ and $\mathbf{M}$ phase of chromonic LCs are known to exhibit negative birefringence, ${ }^{7}$ it can be concluded that the preferred direction of the columnar aggregates (the director) is aligned along the rubbing direction.

The influence and behavior of colloidal particles were tested for three different types of cross-linked PMMA spheres with diameters of about $200 \mathrm{~nm}$ :

Type (A): PMMA spheres functionalized with bromine atoms by copolymerization with bromo hexyl acrylate;

Type (B): positively charged PMMA spheres obtained by substitution of the bromine atoms of type (A)-particles with trimethylamine groups;

Type (C): PMMA spheres functionalized with oxiran groups by copolymerization with glycidyl methacrylate.

The non-charged particles of type (A) tend to aggregate and cannot be dispersed well in the isotropic phase. This lack of dispersibility can probably be attributed to the fact that these particles exhibit the least hydrophilic surface. Since particles of type (A) turned out not to be suitable for our purpose, results on these particles are not shown. However, the charged particles of type (B) and the epoxy-functionalized particles of type (C) disperse very well, at least in the isotropic phase. The dispersion of colloidal particles does not affect the topology of the phase 
diagram and the transition temperatures (Fig. 1) to any great extent.

While the particles of types (B) and (C) disperse homogeneously in the isotropic phase of DSCG mixtures, they tend to form agglomerates at the isotropic to nematic $(\mathrm{I} \rightarrow \mathrm{N})$ and the isotropic to columnar $(\mathrm{I} \rightarrow \mathrm{M})$ phase transitions. Both the functionalization of the particles and the kind of phase appearing at low temperatures are found to influence this agglomeration process. Particles of type (B) form agglomerates of random shape (Fig. 2). Focusing on different planes in the microscope indicates that they are assembling at the cell surface and are probably pinned at the surface. The shape and size of the clusters look to be similar in the $\mathrm{N}$ and $\mathrm{M}$ phases. The top images of Fig. 2 show the liquid crystal textures observed with crossed polarizers at two different temperatures, while the lower images show the sample under the same conditions without polarizers. In the latter case, the agglomerates can be seen very clearly. Comparison of the upper and lower images reveals that the director alignment and thus the birefringence show distinct changes in the vicinity of the agglomerates.

In contrast, the agglomeration of particles of type (C) at the transition from the isotropic to the nematic phase $(\mathrm{I} \rightarrow \mathrm{N})$ appears quite different from the behavior at the isotropic to columnar $(\mathrm{I} \rightarrow \mathrm{M})$ transition (Fig. 3). At the $(\mathrm{I} \rightarrow \mathrm{N})$ transition there is a broad temperature range of $(\mathrm{I}+\mathrm{N})$ coexistence with nematic islands in an isotropic environment; the particles form clusters of irregular shape [Fig. 3(a)]. However, in the (I+M) coexistence range, the growing $\mathrm{M}$ phase forms broad parallel ribbons aligned along the rubbing direction, which are separated by elongated parallel isotropic channels [Fig. 3(b)]. In this environment, the particles are expelled from the $\mathrm{M}$ phase and assemble in the isotropic regions, thereby agglomerating in parallel chains. A sample of $25 \%$ (by weight) DSCG in water shows coexistence of the $\mathrm{N}$ and $\mathrm{M}$ phases at lower temperature
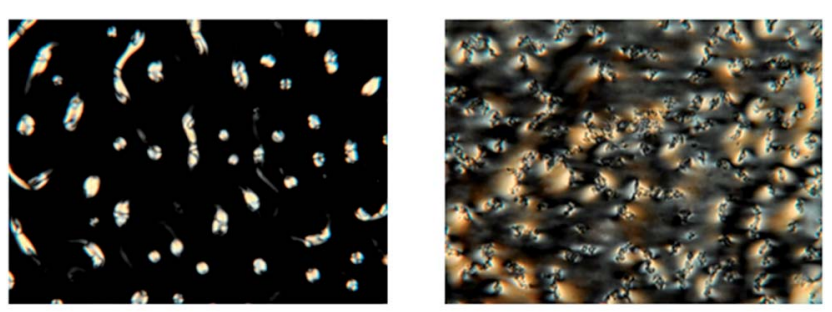

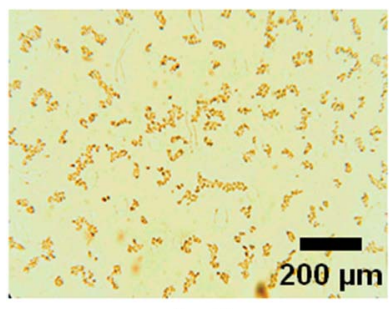

(a)

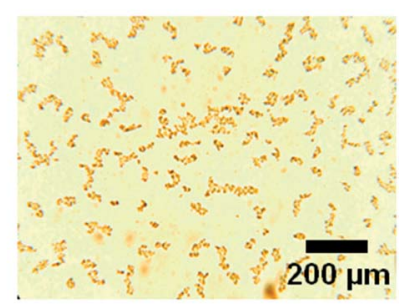

(b)
Fig. 2 Textures of a sample containing 12\% (by weight) DSCG in water and $0.5 \%$ of positively charged PMMA spheres [type (B)] with crossed polarizers (top) and without polarizers (bottom). (a) $30.0^{\circ} \mathrm{C}$ : coexistence of I- and $\mathrm{N}$-phase, (b) $20.0^{\circ} \mathrm{C}$ : N-phase.

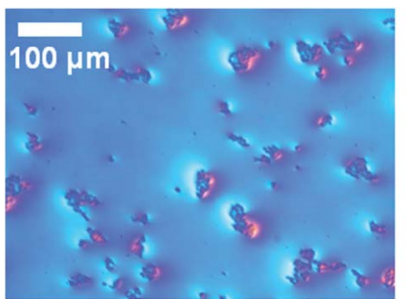

(a)

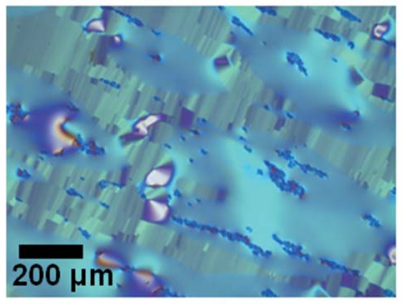

(c)

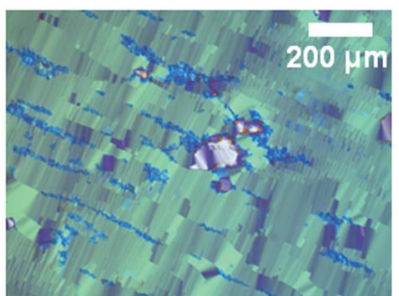

(b)

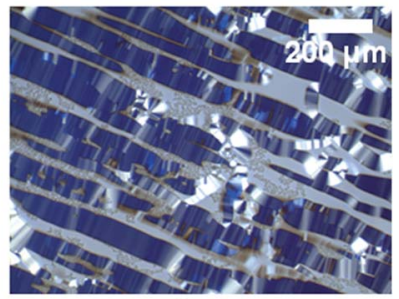

(d)
Fig. 3 Textures of samples containing 4.9\% of epoxy-functionalized PMMA spheres [type (C)] observed with $(\mathrm{a}-\mathrm{c}$ ) crossed polarizers and (d) polarizers at $45^{\circ}$ to one another. (a) $C_{D S C G}=15 \mathrm{wt} \%, T=30.0^{\circ} \mathrm{C}: \mathrm{N}-$ phase, (b) $C_{D S C G}=25 \mathrm{wt} \%, T=44.9^{\circ} \mathrm{C}$ : coexistence of $\mathrm{I}-$ and $\mathrm{M}$ phases, (c) $C_{D S C G}=25 \mathrm{wt} \%, T=41.5^{\circ} \mathrm{C}$ : coexistence of $\mathrm{N}$ - and $\mathrm{M}$ phases, (d) $C_{\mathrm{DSCG}}=25 \mathrm{wt} \%, T=44.9^{\circ} \mathrm{C}$ : coexistence of $\mathrm{I}-$ and $\mathrm{M}$ phases.

[Fig. 3(c)]. The regions of the $\mathrm{N}$ phase show a kind of Schlieren texture with a continuous variation between different grey shades, while the texture of the $\mathrm{M}$ phase shows characteristic sharp grain boundaries, which appear also in the ribbons of the $M$ phase in the $(\mathrm{I}+\mathrm{M})$ coexistence region. Inspection of the colloidal particle distribution in the $(\mathrm{N}+\mathrm{M})$ coexistence region [Fig. 3(c)] shows that agglomerates of arbitrary shape can be formed in the nematic state, while the particles are completely expelled from the regions of the $\mathrm{M}$ phase and tend to assemble at the surface of $\mathrm{M}$ phase regions. Obviously, this expelling of colloidal particles from the $\mathbf{M}$ phase is also driving the arrangement of particles in the $(\mathrm{I}+\mathrm{M})$ coexistence range [Fig. 3(b) and (d)]. When the polarizers of the microscope are not crossed, but aligned at $45^{\circ}$ to each other, one can see both the birefringence of the liquid crystal and the location of the particle agglomerates very clearly [Fig. 3(d)]. Obviously, the particles form long, elongated agglomerates because they are expelled from the parallel ribbons of the $\mathrm{M}$ phase and remain in the channels of isotropic phase. The parallel ordering of the ribbons in turn can be attributed to the surface treatment of the substrate by parallel rubbing with the abrasive foam.

\section{Discussion}

The phenomenon that colloidal particles or nanoparticles are expelled from a mesophase has also been observed at nematic/ isotropic interfaces of thermotropic liquid crystals. ${ }^{23-25}$ It is wellknown that surface effects and elastic deformations of the LC structure affect the free energy density and yield forces acting on defects or enclosed particles in a liquid crystal. The local 
alignment of rod-like liquid crystal molecules (in thermotropic LC) or rod-like molecular aggregates (in lyotropic LC) can be described by a pseudo-vector, the director $\mathbf{n}$, which may depend on the position. A deformation of the director field leads to an increase of the free energy. ${ }^{1}$

$$
\begin{gathered}
f=1 / 2 \int\left[K_{11}(\operatorname{div} \mathbf{n})^{2}+K_{22}(\mathbf{n} \cdot \operatorname{rot} \mathbf{n})^{2}+K_{33}(\mathbf{n} \times \operatorname{rot} \mathbf{n})^{2}\right] \mathrm{dV} \\
+1 / 2 \int\left[W_{\varphi} \sin ^{2}\left(\varphi-\varphi_{0}\right)+W_{\vartheta} \sin ^{2}\left(\vartheta-\vartheta_{0}\right)\right] \mathrm{d} A .
\end{gathered}
$$

The volume integral represents the Frank-Oseen energy describing splay $\left(K_{11}\right)$ twist $\left(K_{22}\right)$ and bend $\left(K_{33}\right)$ deformations of the director, while the surface integral introduced by Rapini and Papoular describes the azimuthal $\left(W_{\varphi}\right)$ and polar $\left(W_{\vartheta}\right)$ anchoring strength of the director at the interface. ${ }^{1}$ According to a model developed by West et al., ${ }^{23,24}$ a dragging force $F_{\text {drag }}$ acts on particles at the nematic-isotropic interface. This force $F_{\mathrm{drag}}$ $=F_{\mathrm{b}}+F_{\mathrm{S}}+F_{\sigma}+F_{\eta}$ is governed by bulk and surface terms of the elastic energy $F_{\mathrm{b}}$ and $F_{\mathrm{s}}$, a contribution $F_{\sigma}$ from the difference of surface tensions at the particle/nematic and particle/isotropic phase interface, and a friction force $F_{\eta}$ originating from the viscous drag. For weak anchoring $(W R / K \ll 1)$ and particles with a radius $R$, the dragging force is given by

$$
F_{\text {drag, weak anchoring }}=\gamma_{\mathrm{b}} W^{2} R^{2} / K+\gamma_{\mathrm{s}} W R+2 \pi R \Delta \sigma-6 \pi R \eta v,(2)
$$

where $W$ and $K$ are effective values of the anchoring energy and the elastic coefficients, $\gamma_{b}$ and $\gamma_{s}$ are dimensionless geometrical parameters, $\Delta \sigma$ is the difference of the surface tensions in the mesophase and in the isotropic phase, $\eta$ is the effective viscosity and $v$ the velocity at which the phase boundary propagates. Since the elastic force scales as $R^{2}$ while the friction force scales as $R$, particles move only if their radius $R$ exceeds a minimum value $R_{\min }$, which is given by the condition $F_{\text {drag, weak anchoring }}=$ 0 in eqn (2). On the other hand, the moving phase boundary can transfer only a limited momentum to a particle, which limits the particle size to a condition $R<R_{\max }(v, W, \Delta \sigma)$. For strong anchoring $(W R / K \gg 1)$, the dragging force is given by ${ }^{23}$

$$
F_{\text {drag, strong anchoring }}=\gamma_{\mathrm{b}} K+2 \pi R \Delta \sigma-6 \pi R \eta v \text {. }
$$

If the particles expelled from the mesophase start agglomerating, the effective particle size $R$ no longer corresponds to the radius of a single particle, but to the size of an agglomerate of particles. Thus, agglomeration may cause a transition from weak anchoring $(W R / K \ll 1)$ to strong anchoring $(W R / K \gg 1)$ conditions. In the strong anchoring regime, a critical size of agglomerates $R_{\text {crit }}$ [given by the condition $F_{\text {drag, strong anchoring }}=$ 0 in eqn (3)] may be exceeded, where chains of particles break through the phase boundary and remain in the mesophase. Studies of colloidal particles in a thermotropic nematic liquid crystal $(5 \mathrm{CB})$ with $K \approx 10^{-12}-10^{-11} \mathrm{~N}, W \approx 10^{-7}-10^{-6} \mathrm{~N} \mathrm{~m}^{-1}$ and $\Delta \sigma \approx 10^{-4}-10^{-3} \mathrm{~N} \mathrm{~m}^{-2}$ have revealed that small silica particles $(R \approx 5 \mathrm{~nm}-500 \mathrm{~nm})$ represent weak anchoring conditions, while large polymer particles $(R \approx 8 \mu \mathrm{m})$ and sufficiently large agglomerates of small particles represent the strong anchoring regime. ${ }^{23}$ Both kinds of particles can be dragged by an interface moving at the velocity $v \approx 3 \mu \mathrm{m} \mathrm{s}^{-1}$ and are expelled from the nematic phase.
The experiments reported here indicate that PMMA particles with a size similar to the range of silica particles that were studied in ref. 23 and 24 are not expelled by the chromonic nematic $(\mathrm{N})$ phase, but can be expelled by the chromonic columnar (M) phase, thereby forming a stripe pattern, which resembles the stripe pattern shown in ref. 23. This observation may be explained by the dragging model taking into account the elastic coefficients of chromonic liquid crystals. Measurements of the elastic coefficients of the lyotropic chromonic nematic phase formed by solutions of the dye sunset yellow (SSY) ${ }^{26}$ have shown that the splay and bend elastic coefficients $K_{11}$ and $K_{33}$ exhibit similar size to those of thermotropic nematic liquid crystals, while the value of $K_{22}$ is about one order of magnitude smaller in the chromonic $\mathrm{N}$ phase. Recent studies of the director field around microparticles dispersed in a chromonic $\mathrm{N}$ phase revealed an unusual twisted director field around these particles even in non-chiral systems, which can be attributed to the unusually small value of the twist elastic coefficient $K_{22}{ }^{27}$ This ability to accommodate particle inclusion by lowering the elastic energy through spontaneous twist, thus decreasing the average elastic constant, may explain why the particles in our samples are not observed to be expelled by the chromonic nematic phase. On the other hand, it is known that a columnar phase can not be splayed or twisted without the appearance of edge- or screw dislocations, respectively. ${ }^{1}$ Therefore, we expect the splay and twist coefficients $K_{11}$ and $K_{22}$ to diverge at the transition to the columnar phase. If the elastic coefficients of the chromonic columnar phase are equal to or larger than the elastic coefficients of a thermotropic nematic phase, the particles are expected to be dragged at the interface and to assemble in the isotropic phase, as observed in the present study.

\section{Experimental}

\section{Particle synthesis}

Cross-linked, functionalized colloidal particles (Table 1) were synthesized by surfactant-free radical polymerization ${ }^{28}$ of methyl methacrylate (MMA) with the cross-linking agent dimethyl 2,2'-[oxybis(methylene)] bisacrylate ${ }^{29,30}$ and different monofunctional co-monomers (Fig. 4). The cross-linker was synthesized as described in ref. 29. 6-Bromohexyl acrylate was used as a co-monomer to obtain particles functionalized with bromine atoms [type (A)] and positively charged particles [type (B)]. ${ }^{31}$ The monomers were destabilized before use by flash column chromatography using basic aluminum oxide as the solid phase. For the particle synthesis, $140 \mathrm{ml}$ of distilled water was heated to $80{ }^{\circ} \mathrm{C}$ and degassed with argon. A mixture of the respective monomers (Table 1) with $15 \mathrm{ml}$ toluene was added and the resulting mixture was vigorously stirred and annealed at $80{ }^{\circ} \mathrm{C}$ before the reaction was started by adding a solution of $0.8 \mathrm{~g} \mathrm{~K}_{2} \mathrm{~S}_{2} \mathrm{O}_{7}$ in $20 \mathrm{ml}$ degassed water. Stopping the reaction after 20 minutes by fumigating with air and cooling, evaporation of the residual monomer and of toluene, and subsequent centrifugation and purification of the particles yielded monodisperse particles of type (A) with a diameter of about $180 \mathrm{~nm}$ (determined by Zetasizer, Malvern). For getting positively charged particles, quaternary ammonium groups with bromide 
Table 1 Monomer composition of the functionalized particles

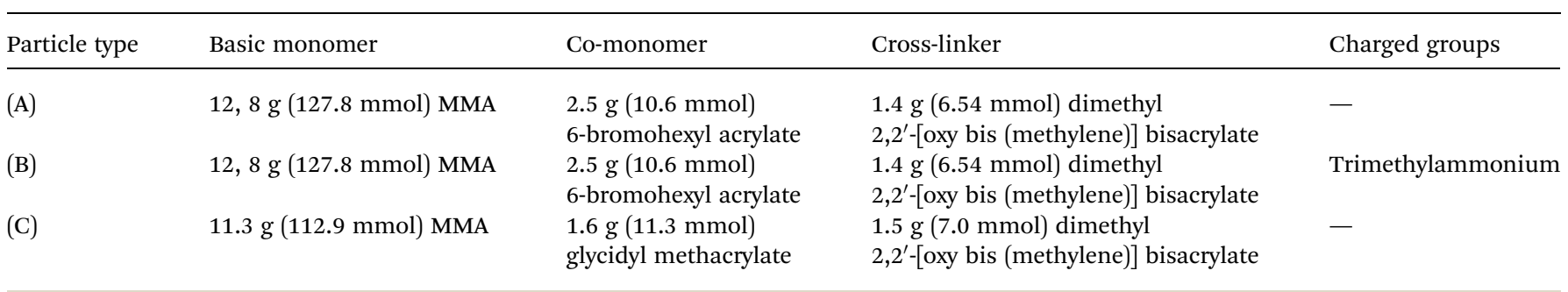

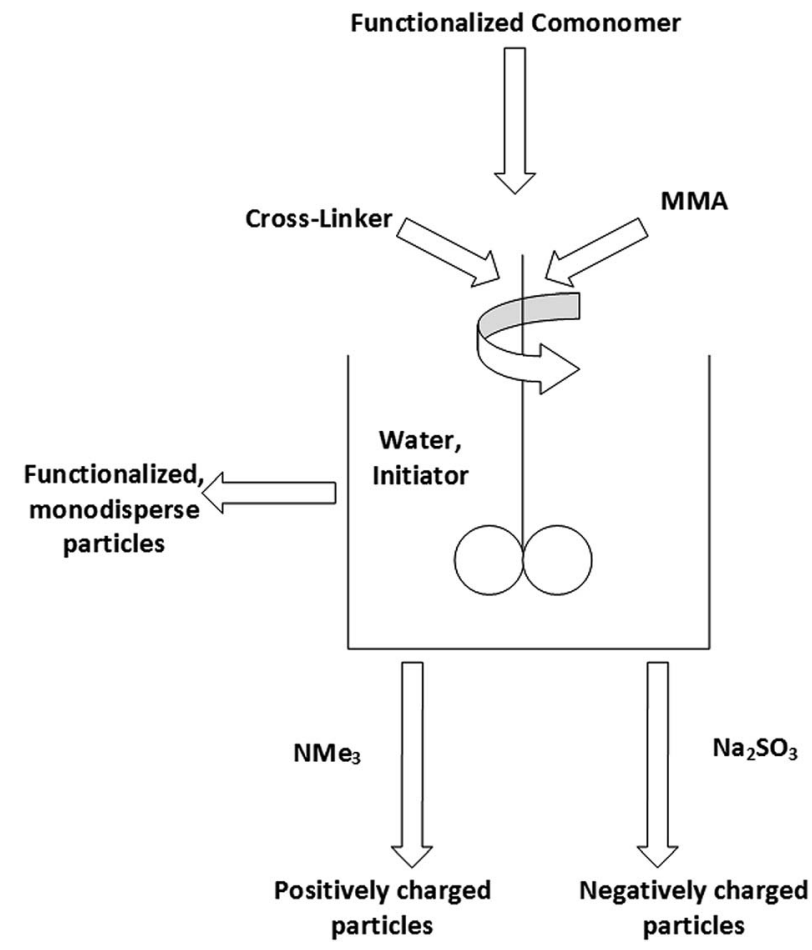

Fig. 4 Flow-diagram of surfactant-free polymerization with bromo hexyl acrylate (yielding positively or negatively charged PMMA particles after substitution of the bromine atoms).

counter-ions were generated through substituting the bromo functionality by trimethylamine. For this purpose, an aqueous dispersion of type (A) particles was stirred with an excess of trimethylamine for 72 hours at room temperature. The resulting particles of type (B) were separated by centrifugation. Epoxyfunctionalized particles [type (C)] were synthesized using glycidyl methacrylate as a co-monomer as described in ref. 32 . Stopping the polymerization reaction after 15 minutes yielded particles with a diameter of 160-170 nm (determined by Zetasizer, Malvern). The surface charge density of the particles (up to $90 \mu \mathrm{C} \mathrm{cm}{ }^{-2}$ ) and its measurement were extensively described in a previous paper (ref. 31).

\section{Cell preparation and characterization}

DSCG was purchased from Sigma-Aldrich and used without further purification for preparing mixtures with 12.5 to 30.0 $\mathrm{wt} \%$ of DSCG in millipore water. For preparing cells,

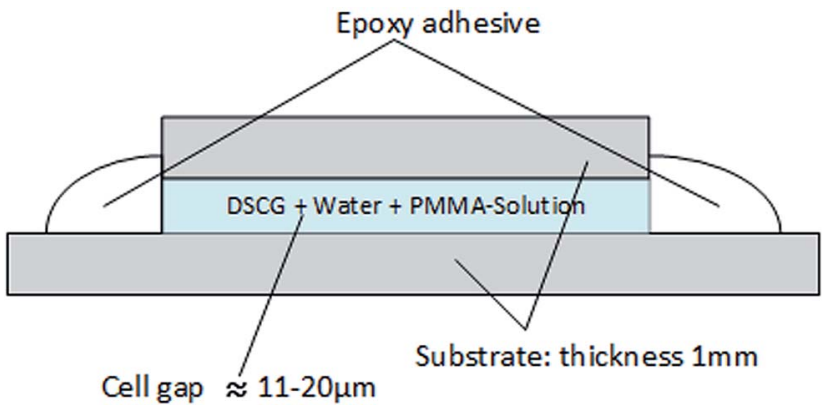

Fig. 5 Cell geometry.

microscope slides were cleaned with acetone and millipore water and subsequently exposed to plasma cleaning. Alignment of the director on the glass surfaces was achieved by rubbing the microscope slide once with a fine abrasive foam (3M Trizact ${ }^{\mathrm{TM}}$ Foam Disc P3000) under pressure. ${ }^{\mathbf{1 4}}$ From these prepared microscope slides, cells with a gap of $11 \mu \mathrm{m}$ to $20 \mu \mathrm{m}$ were fabricated [Fig. 5]. The cell gap was controlled by dispersing glass cylinders with a well defined diameter in two-component epoxy glue, which was used to fix the slides. When isotropic DSCG solutions are filled into an $11 \mu \mathrm{m}$ cell with parallel rubbing directions at elevated temperature and subsequently cooled slowly, an oriented single domain of the $\mathrm{N}$ or oriented domains of the $\mathbf{M}$ phase are obtained. The cells were investigated by a polarizing microscope (model DM 4500 P, Leica), while controlling the temperature with a FP82 hot stage addressed by a FP 90 controller (Mettler).

\section{Conclusion}

In summary, the stability of different colloidal particles in DSCG/water-based chromonic liquid crystals turned out to depend on the surface functionality of the particles. While noncharged particles functionalized with bromine atoms do not disperse in the liquid crystal, cationic trimethylammoniumsubstituted PMMA particles and epoxy-functionalized PMMA particles disperse very well in the isotropic phase of the DSCG/ water mixtures and agglomerate only in the liquid crystalline phases. When respective mixtures are cooled from the isotropic phase, this agglomeration starts in the temperature range where the isotropic phase (I) and the $\mathrm{N}$ or M mesophase coexist. Particularly in the case of $(I+M)$ coexistence, it is obvious that the particles are completely expelled from the $\mathbf{M}$ phase and 
therefore assemble in the I phase. Surface alignment induced by parallel rubbing of the glass substrates with an abrasive prior to filling the cells yields not only uniform alignment of the optical axis of the $\mathrm{N}$ phase, but also the growth of parallel ribbons of the $\mathbf{M}$ phase, which are separated by parallel channels of the I phase. This spontaneous pattern formation in turn leads to the formation of stripe-like assemblies of the particles in the isotropic phase.

The phenomenon that colloidal particles or nanoparticles are expelled from a mesophase has been previously observed at the nematic/isotropic interface of thermotropic liquid crystals and can be explained by dragging forces acting on the particles at the isotropic/mesophase interface, which are caused by director field distortions in the mesophase and differences of the surface tensions and may be compensated by viscous drag. ${ }^{23,24}$ Our observation that nanoparticles are expelled by the chromonic columnar phase is in agreement with the model by West et al. ${ }^{23,24}$ developed for thermotropic mesophases. The finding that the particles are not expelled from the chromonic nematic phase may be explained by the unusually small value of the twist constant $K_{22}$, which is known to be one order of magnitude lower in the chromonic nematic phase than in a thermotropic nematic phase $\mathrm{e}^{26}$ thereby easing the inclusion of particles. ${ }^{27}$ More quantitative results may be obtained by detailed studies of the director field around colloidal particles, for example by fluorescence confocal polarizing microscopy (FCPM) ${ }^{33-36}$ Such detailed studies - which are beyond the scope of the present paper - will be the subject of forthcoming investigations.

\section{Acknowledgements}

The authors would like to thank Marina Kley and Klaus Huber for their assistance in measuring the particle sizes by means of dynamic light scattering. Financial support by the Alexandervon-Humboldt-Foundation and the Deutsche Forschungsgemeinschaft (GRK 1464) is gratefully acknowledged.

\section{Notes and references}

1 P. G. de Gennes and J. Prost, The Physics of Liquid Crystals, Clarendon Press, Oxford, 1993.

2 Handbook of Liquid Crystals, ed. J. W. Goodby, P. J. Collings, H. Gleeson, T. Kato, P. Raynes and C. Tschierske, Wiley-VCH, Weinheim, 2014.

3 J. Lydon, Chromonic liquid crystalline phases, Liq. Cryst., 2011, 38, 1663-1681.

4 J. Lydon, Chromonic review, J. Mater. Chem., 2010, 20, 10071-10099.

5 P. J. Collings, A. J. Dickinson and E. C. Smith, Molecular aggregation and chromonic liquid crystals, Liq. Cryst., 2010, 37, 701-710.

6 C. J. Woolverton, E. Gustely, L. Li and O. D. Lavrentovich, Liquid crystal effects on bacterial viability, Liq. Cryst., 2005, 32, 417-423.

7 V. R. Horowitz, L. A. Janowitz, A. L. Modic, P. A. Heiney and P. J. Collings, Aggregation behavior and chromonic liquid crystal properties of an anionic monoazo dye, Phys. Rev. E: Stat., Nonlinear, Soft Matter Phys., 2005, 72, 041710.

8 A. Miyatake, M. Fujita, Y. Nagasaka, K. Fujita, M. Tamari, D. Watanabe, N. Nakano, K. I. P. J. Hidari and Y. Suzuki, The New Role of Disodium Cromoglycate in the Treatment of Adults with Bronchial Asthma, Allergol. Int., 2007, 56, 231-239.

9 K. A. Simon, P. Sejwal, E. R. Falcone, E. A. Burton, S. Yang, D. Prashar, D. Bandyopadhyay, S. K. Narasimhan, N. Varghese, N. S. Gobalasingham, J. B. Reese and Y.-Y. Luk, Noncovalent Polymerization and Assembly in Water Promoted by Thermodynamic Incompatibility, $J$. Phys. Chem. B, 2010, 114(32), 10357-10367.

10 S. V. Shiyanovskii, T. Schneider, I. I. Smalyukh, T. Ishikawa, G. D. Niehaus, K. J. Doane, C. J. Woolverton and O. D. Lavrentovich, Real-time microbe detection based on director distortions around growing immune complexes in lyotropic chromonic liquid crystals, Phys. Rev. E: Stat., Nonlinear, Soft Matter Phys., 2005, 71, 020702.

11 V. G. Nazarenko, O. P. Boiko, M. I. Anisimov, A. K. Kadashchuk, Y. A. Nastishin, A. B. Golovin and O. D. Lavrentovich, Lyotropic chromonic liquid crystal semiconductors for water-solution processable organic electronics, Appl. Phys. Lett., 2010, 97, 263305.

12 F. Guo, A. Mukhopadhyay, B. W. Sheldon and R. H. Hurt, Vertically Aligned Graphene Layer Arrays from Chromonic Liquid Crystal Precursors, Adv. Mater., 2011, 23, 508-513.

13 V. G. Nazarenko, O. P. Boiko, H.-S. Park, O. M. Brodyn, M. M. Omelchenko, L. Tortora, Y. A. Nastishin and O. D. Lavrentovich, Surface Alignment and Anchoring Transitions in Nematic Lyotropic Chromonic Liquid Crystal, Phys. Rev. Lett., 2010, 105, 017801.

14 C. K. McGinn, L. I. Laderman, N. Zimmermann, H.-S. Kitzerow and P. J. Collings, Phys. Rev. E: Stat., Nonlinear, Soft Matter Phys., 2013, 88, 062513.

15 Y. Yi and N. A. Clark, Orientation of chromonic liquid crystals by topographic linear channels: multi-stable alignment and tactoid structure, Liq. Cryst., 2013, 40(12), 1736-1747.

16 Y. Xia, B. Gates, Y. Yin and Y. Lu, Monodispersed Colloidal Spheres: Old Materials with New Applications, Adv. Mater., 2000, 12, 603-713.

17 N. H. Hartshorne and G. D. Woodard, Mesomorphism in the system disodium chromoglycate-water, Mol. Cryst. Liq. Cryst., 1973, 23, 343.

18 D. M. Agra-Kooijman, G. Singh, A. Lorenz, P. J. Collings, H. S. Kitzerow and S. Kumar, Columnar Molecular Aggregation in the Aqueous Solutions of Disodium Cromoglycate, Phys. Rev. E: Stat., Nonlinear, Soft Matter Phys., 2014, 89, 062504.

19 J. Lydon, New Models for the Mesophases of Disodium Cromoglycate (INTAL), Mol. Cryst. Liq. Cryst., 1980, 64, 19-24.

20 L. Wu, J. Lal, K. Simon, E. Burton and Y.-Y. Luk, Nonamphiphilic Assembly in Water: Polymorphic Nature, Thread Structure, and Thermodynamic Incompatibility, $J$. Am. Chem. Soc., 2009, 131, 7430-7443. 
21 L. Tortora, H.-S. Park, S.-W. Kang, V. Savaryn, S.-H. Hong, K. Kaznatcheev, D. Finotello, S. Sprunt, S. Kumar and O. D. Lavrentovich, Self-assembly, condensation, and order in aqueous lyotropic chromonic liquid crystals crowded with additives, Soft Matter, 2010, 6, 4157-4167.

22 M. Gao, Y.-K. Kim, C. Zhang, V. Borshch, S. Zhou, H.-S. Park, A. Jákli, O. D. Lavrentovich, M.-G. Tamba, A. Kohlmeier, G. H. Mehl, W. Weissflog, D. Studer, B. Zuber, H. Gnägi and F. Lin, Direct observation of liquid crystals using cryoTEM: Specimen preparation and low-dose imaging, Microsc. Res. Tech., 2014, 77(10), 754-772.

23 J. L. West, A. Glushchenko, G. Liao, Y. Reznikov, D. Andrienko and M. P. Allen, Drag on particles in a nematic suspension by a moving nematic-isotropic interface, Phys. Rev. E: Stat., Nonlinear, Soft Matter Phys., 2002, 66, 012702.

24 J. L. West, K. Zhang, G. Liao, A. V. Glushchenko, Y. Reznikov, D. Andrienko and M. P. Allen, Mechanism of Formation of Three Dimensional Structures of Particles in a Liquid Crystal, Mol. Cryst. Liq. Cryst., 2004, 410(1), 83-93.

25 J. L. West, K. Zhang, A. Glushchenko, D. Andrienko, M. Tasinkevych and Y. Reznikov, Colloidal particles at a nematic-isotropic interface: Effects of confinement, Eur. Phys. J. E: Soft Matter Biol. Phys., 2006, 20(2), 237-242.

26 S. Zhou, Y. A. Nastishin, M. M. Omelchenko, L. Tortora, V. G. Nazarenko, O. P. Boiko, T. Ostapenko, T. Hu, C. C. Almasan, S. N. Sprunt, J. T. Gleeson and O. D. Lavrentovich, Elasticity of Lyotropic Chromonic Liquid Crystals Probed by Director Reorientation in a Magnetic Field, Phys. Rev. Lett., 2012, 109, 037801.

27 A. Nych, U. Ognysta, I. Muševič, D. Seč, M. Ravnik and S. Žumer, Chiral bipolar colloids from nonchiral chromonic liquid crystals, Phys. Rev. E: Stat., Nonlinear, Soft Matter Phys., 2014, 89, 062502.
28 M. Müller, R. Zentel, T. Maka, S. G. Romanov and C. M. Sotomayor Torres, Dye-Containing Polymer Beads as Photonic Crystals, Chem. Mater., 2000, 12, 2508-2512.

29 S. E. Drewes, G. Loizou and G. H. P. Roos, A Facile, Efficient Route to Methyl $\alpha$-Hydroxymethylacrylate and Methyl $\alpha$ Halomethylacrylates, Synth. Commun., 1987, 17(3), 291-298.

30 U. Flörke, T. Röder and T. Kramer, Methyl 4-oxahepta-1,6diene-2,6-dicarboxylate, Acta Crystallogr., Sect. E: Struct. Rep. Online, 2002, 58, o1343-01344.

31 T. Röder, T. Kramer, K. Huber and H.-S. Kitzerow, Preparation of Positively and Negatively Charged Organic Colloids from a Single Precursor, Macromol. Chem. Phys., 2003, 204, 2204-2211.

32 T. Kramer, T. Röder, K. Huber and H.-S. Kitzerow, Surface modification of epoxy-functionalized acrylate colloids, Polym. Adv. Technol., 2005, 16, 38-41.

33 I. I. Smalyukh, S. V. Shivanovskii and O. D. Lavrentovich, Three-dimensional imaging of orientational order by fluorescence confocal polarizing microscopy, Chem. Phys. Lett., 2001, 336(1-2), 88-96.

34 I. I. Smalyukh and O. D. Lavrentovich, Three-dimensional director structures of defects in Grandjean-Cano wedges of cholesteric liquid crystals studied by fluorescence confocal polarizing microscopy, Phys. Rev. E: Stat., Nonlinear, Soft Matter Phys., 2002, 66, 051703.

35 M. Urbanski, K. A. Piegdon, C. Meier and H.-S. Kitzerow, Investigations on the director field around microdisk resonators, Liq. Cryst., 2011, 38(4), 475-482.

36 M. Urbanski, J. Mirzaei, T. Hegmann and H.-S. Kitzerow, Nanoparticle Doping in Nematic Liquid Crystals: Distinction between Surface and Bulk Effects by Numerical Simulations, ChemPhysChem, 2014, 15, 1395-1404. 